\title{
Hofzeremoniell und Gebärstuhl. Zur Bedeutung von Ritualen bei den Entbindungen Maria Theresias (1717-1780)
}

\author{
Sabrina Schober \\ Kerngebiet: Wirtschafts- und Sozialgeschichte \\ eingereicht bei: Mag. Dr. Marina Lucy Hilber, Bakk. \\ eingereicht im: WiSe 2019/20 \\ Rubrik: Seminar-Arbeit (Vertiefung)
}

\begin{abstract}
Ceremonial and a birthing chair. The significance of rituals during the childbirths of Maria Theresia (1717-1780)

Maria Theresia (1717-1780), Archduchess of Austria, Queen of Bohemia and Hungary, delivered sixteen children in twenty years. The following seminar paper aims to reconstruct the deliveries of Maria Theresia in order to give an insight into childbirths of royals in the 18th century. It covers the childbirth itself as well as the following activities to understand the importance of rituals and ceremonials surrounding the deliveries, considering that children were equivalent to the continuity of the monarchy and the Habsburg dynasty. To this end, the paper analyses letters written by Maria Theresia herself as well as records of her master of ceremonies.
\end{abstract}

\section{Einleitung}

„Nehmen sie sich kein Beispiel an mir, denn ich war immer nur zu glücklich bei meinen Entbindungen, mehr als ich es verdiente.." - Maria Theresia an ihre Schwester.

Friedrich Walter (Hrsg.), Maria Theresia. Briefe und Aktenstücke in Auswahl (Freiherr-vom-Stein-Gedächtnisausgabe. Ausgewählte Quellen zur Deutschen Geschichte der Neuzeit 12), Darmstadt 1968, S. 39-49, zit. nach Barbara Stollberg-Rilinger, Maria Theresia. Die Kaiserin in ihrer Zeit, München 2017, S. 295. 
Die Hauptaufgabe einer Habsburgerin und wohl jeder anderen Frau gehobenen Standes im 18. Jahrhundert war das Gebären von Kindern. Das Fortbestehen der jeweiligen Dynastie sollte dadurch gesichert werden. Dabei galt: je mehr Kinder, desto besser. Aufgrund der hohen Säuglings- und Kindersterblichkeit sowie Krankheiten - zur Zeit Maria Theresias vor allem die Pocken - musste jederzeit mit dem Schlimmsten gerechnet werden. Maria Theresia wurde dieser Aufgabe vollends gerecht. Ein Jahr nach ihrer Hochzeit mit Franz Stephan von Lothringen (1708-1765) gebar sie im Alter von 19 Jahren ihr erstes Kind. Bis zu ihrem vierzigsten Lebensjahr folgten 15 weitere Entbindungen. Von den insgesamt 16 Kindern des Kaiserpaares sollten zehn das Erwachsenenalter erreichen. Die Anzahl der Schwangerschaften war für die damalige Zeit nicht außergewöhnlich. Besonders war, dass der Großteil der Nachkommenschaft die Kinderjahre überlebte. Das Mutterbild Maria Theresias hielt sich wie kein anderer Topos rund um die Herrscherin. Ihr biologischer weiblicher Körper ermöglichte die Metapher der Mutter des Volkes. ${ }^{2}$

Die Erforschung der Rituale bei den Entbindungen Maria Theresias steht im Zentrum dieser Arbeit. Dabei werden folgende Fragen aufgeworfen: Welche Personen waren während der Entbindungen der Herrscherin anwesend und welche Rituale sind im Umfeld der Entbindungen erkennbar? Die leitende These dabei lautet: Die Schwangerschaften Maria Theresias unterlagen einer bestimmten Regelung durch das Hofzeremoniell, welches von der Bekanntgabe der Schwangerschaft über die Entbindung bis zur Taufe des Kindes genaue Rituale vorgab.

Maria Theresia stellt als einzige weibliche Thronfolgerin im Hause Habsburg eine Besonderheit dar, was sich auch in Publikationen widerspiegelt: Ihre Person wurde über die Jahrhunderte hinweg immer wieder zum Gegenstand geschichts- sowie populärwissenschaftlicher Werke. Großteils handelt es sich dabei um politische Biographien, Veröffentlichungen ihrer Briefe und Ähnliches. Anlässlich des dreihundertsten Geburtstags Maria Theresias im Jahre 2017 kam es zu einer Welle an wissenschaftlichen Publikationen. Eine neue Perspektive auf die Erzherzogin eröffnete die Historikerin Barbara Stollberg-Rilinger mit ihrer Biographie „Maria Theresia. Die Kaiserin in ihrer Zeit“3. Laut einer Rezension sei diese die erste ernstzunehmende wissenschaftliche Biographie zu Maria Theresia seit dem Ende der Monarchie. ${ }^{4}$ Getreu ihren Forschungsschwerpunkten erörtert Stollberg-Rilinger in ihrem Werk wiederholt die Rituale im Leben Maria Theresias zur Darstellung ihrer Macht. Aus diesem Grund soll das Werk auch für die vorliegende Arbeit herangezogen werden. Während also zu Maria Theresia selbst sowie deren Nachkommen unzählige Biographien existieren, ist es schwierig, Publikationen explizit zu den Entbindungen - besonders bezogen auf deren Rituale - zu finden. Zur Bearbeitung und Analyse dieser Rituale liefern die Hofzeremonielle und Verlautbarungen der

2 Zu den verschiedenen Körpern Maria Theresias sei hier vor allem auf einen Beitrag von Anne-Sophie Banakas verwiesen: Anne-Sophie Banakas, Die zwei Körper der Herrscherin. Der politische und der natürliche Körper in den Porträts von Maria Theresia (1740-1780), in: Mitteilungen des Instituts für Österreichische Geschichtsforschung 126 (2018), Heft 1, S. 73-109.

3 Barbara Stollberg-Rilinger, Maria Theresia. Die Kaiserin in ihrer Zeit, München 2017.

4 Matthias Schnettger, Rezension zu: Barbara Stollberg-Rilinger, Maria Theresia. Die Kaiserin in ihrer Zeit, München 2017, in: sehepunkte 17 (2017), Heft 9, http://www.sehepunkte.de/2017/09/30136.html, eingesehen 14.2.2020. 
Geburten eine wichtige Quelle. Neben dem bereits genannten Werk sollen auch zwei Werke von Karl Vocelka und Lynne Heller ${ }^{5}$ herangezogen werden, die sich mit der Welt der Habsburger*innen und damit mit deren Ritualen beschäftigen.

Um den Forschungsfragen nachzugehen, werden neben der Sekundärliteratur auch schriftliche Quellen herangezogen. Dabei handelt es sich vor allem um Briefe ${ }^{6}$, die Maria Theresia an ihre Kinder und engsten Vertrauten schrieb und aus dem Französischen übersetzt vorliegen, Zeitungsausschnitte aus der "Wiener Zeitung"7, in denen die Geburten verlautbart wurden sowie Tagebuchaufzeichnungen von Johann Joseph von Khevenhüller-Metsch ${ }^{8}$ (1706-1776), der in verschiedensten hohen Positionen, zuletzt als Obersthofmeister, am Hofe tätig war. Die Arbeit gliedert sich in zwei große Teile. Der erste Teil behandelt die Unterschiede zwischen royalen und anderen Entbindungen sowie Maria Theresias Vorstellungen einer idealen Schwangerschaft. Kurz gestreift wird auch der Aspekt der rituell bestimmten Verlautbarung der Schwangerschaften. Im zweiten Teil der Arbeit wird das Hauptaugenmerk auf die Entbindungen und deren begleitende Faktoren wie Orte, Anwesende und Verlauf gelegt.

Vorab sollte noch der Begriff "Ritual“ geklärt werden. Ein Ritual beschreibt eine Handlung, die durch Standardisierung, Wiederholung, Abfolge und bestimmte Symbole charakterisiert ist und eine gesellschaftliche Wirkung besitzt. Das heißt zum einen, dass Rituale einer bestimmten äußeren Form folgen und diese sich wiederholt, was ein Ritual erwartbar und wiedererkennbar macht. Dies kann entweder schriftlich festgelegt sein oder allein durch das wiederholte Handeln selbst „festgeschrieben“ werden. Zugleich sind Rituale aber nicht starr in einem Konstrukt verankert, sondern können auch verändert werden. Rituale sind zudem symbolisch in dem Sinne, dass sie auf eine soziale Gemeinschaftsordnung verweisen. Die Einhaltung der Rituale diente auch der Bestätigung der hierarchischen Ordnung, eine Nichteinhaltung konnte als Mittel der Kritik eingesetzt werden. Rituale geben also die grundlegenden Werte und Ordnungen einer Gesellschaft wieder und reproduzieren beziehungsweise übermitteln die soziale Wirklichkeit - besonders für Historiker*innen ein wichtiger Forschungsansatz. Die Ritualforschung hatte ihren ersten Höhepunkt in den 1880er-Jahren, zunächst in den Religionswissenschaften sowie in soziologischen und anthropologischen Forschungen. In den Geschichtswissenschaften herrschte die Politik-, Verfassungs- und Sozialgeschichte lange Zeit vor und so traten Rituale erst später in den historischen Diskurs ein, sind mittlerweile aber zu einem wichtigen Teil der (universitären) Lehre geworden. ${ }^{9}$

$5 \quad$ Karl Vocelka/Lynne Heller, Die Lebenswelt der Habsburger. Kultur- und Mentalitätsgeschichte einer Familie, GrazWien-Köln 1997; Karl Vocelka/Lynne Heller, Die private Welt der Habsburger. Leben und Alltag einer Familie, GrazWien-Köln 1998.

6 Siehe dazu: Carl Rothe (Hrsg.), Die Mutter und die Kaiserin. Briefe der Maria Theresia an ihre Kinder und Vertraute, Berlin 1987.

$7 \quad$ Ab 1703 unter Wienerisches Diarium, ab 1780 als Wiener Zeitung publiziert.

8 Sein Tagebuch wurde zwischen 1907 und 1972 in insgesamt acht Bänden im Auftrag der Gesellschaft für Neuere Geschichte Österreichs herausgegeben.

9 Barbara Stollberg-Rilinger, Rituale (Historische Einführungen 16), Frankfurt am Main 2013, S. 9-36. 


\section{Maria Theresia und ihre Zeit}

Im folgenden Kapitel werden zum einen die Schwangerschaften Maria Theresias betrachtet, zum anderen werden aber auch Geburten außerhalb der Adelsgesellschaft beleuchtet. Dies soll die Besonderheiten von adligen Entbindungen hervorheben und einen generellen Einblick in das Entbindungsgeschehen geben. Weiters soll damit aufgezeigt werden, auf welche rituelle Weise Maria Theresia ihre Schwangerschaften verkündete, welche Gewohnheiten bei einer schwangeren Habsburgerin üblich waren und wie sich diese zu verhalten hatte.

\subsection{Entbindungen außerhalb der Adelsschicht}

Alle Lebensabschnitte wurden von Ritualen begleitet, die das Individuum in die Gemeinschaftsordnung einfügten und diese dadurch immer wieder neu bestätigten. Davon waren auch Schwangerschaft und Entbindung nicht ausgenommen. Die Geburt begann im Normalfall damit, dass die Gebärende in die Obhut von Frauen genommen wurde. Dies konnte eine Hebamme sein, eine Nachbarin oder auch eine Verwandte der Gebärenden. Diese Frauen kümmerten sich während der Entbindung und des folgenden Wochenbettes um die werdende Mutter und praktizierten unterschiedlichste Rituale, die meist der "Schadensabwehr" dienten. Dass männliche Ärzte an der Geburt teilnahmen, setzte in Europa erst ab dem 18. Jahrhundert langsam ein. ${ }^{10}$ Im Zusammenhang mit den im 18. Jahrhundert stattfindenden Modernisierungen im Medizinbereich wollte die Ärzteschaft auch die Ausbildung von Hebammen reformieren. Sie sollten in speziellen Schulen - geführt von männlichen Ärzten - ausgebildet und nicht mehr praktisch angelernt werden, wie zuvor üblich. Dadurch erhoffte man sich eine bessere Ausbildung der Geburtshelferinnen und zugleich die Kontrolle derer. ${ }^{11} 1711$ wurde dementsprechend eine Dienstordnung für Hebammen in Wien erlassen. Diese verpflichtete die Geburtshelferinnen unter anderem zu Gehorsam gegenüber dem Dekan der medizinischen Fakultät, wo sie ihre Ausbildung und Prüfung abzulegen hatten. Ab 1754 durften Hebammen selbst keine Schülerinnen mehr ausbilden. ${ }^{12}$

Die Entbindung in Spitälern beziehungsweise Gebärhäusern war im 18. Jahrhundert eine Seltenheit. Diese waren meist Anlaufstellen für ledige Frauen. Da solche Einrichtungen vor allem dem Unterrichtszweck dienten, war eine Entbindung dort aufgrund der vielen Anwesenden sehr öffentlich und wurde von Frauen wohl kaum freiwillig gewählt.13 Auf österreichischem Gebiet nahm die Stadt Wien eine Vorreiterrolle in Bezug auf Gebärhausgründungen ein. Schon zu Beginn des 18. Jahrhunderts existierte

10 Stollberg-Rilinger, Rituale, S. 55-61.

11 Gunda Barth-Scalmani, „Freundschaftlicher Zuruf eines Arztes an das Salzburgische Landvolk“. Staatliche Hebammenausbildung und medizinische Volksaufklärung am Ende des 18. Jahrhunderts, in: Jürgen Schlumbohm u. a. (Hrsg.), Rituale der Geburt. Eine Kulturgeschichte, München 1998, S. 102-118, hier S. 103.

12 Sonia Horn, „... . dann mit meiner Hebammerey ich vill mehr gewinnen kann, alß mein Mann mit seiner Doctorey.." Wiener Hebammen 1700-1750, Dipl. Wien 1995, S. 60-66.

13 Verena Pawlowsky, Trinkgelder, Privatarbeiten, Schleichhandel mit Ammen. Personal und Patientinnen in der inoffiziellen Ökonomie des Wiener Gebärhauses (1784-1908), in: Jürgen Schlumbohm u. a. (Hrsg.), Rituale der Geburt. Eine Kulturgeschichte, München 1998, S. 206-220, hier S. 206. 
im St. Marxer Spital eine eigene Gebärabteilung. ${ }^{14}$ Hier mussten Hebammen ab 1721 eine gewisse Zeit lang nach ihrer Ausbildung arbeiten, ab 1754 fand vermutlich die gesamte Hebammenausbildung dort statt. ${ }^{15}$

Im Normalfall fanden Entbindungen bei der Gebärenden zuhause statt und waren reine Frauensache. Zu bedenken ist, dass bei Entbindungen regionale Unterschiede auftraten und bestimmte Anwesende sowie der Ablauf von deren Verfügbarkeit beziehungsweise von der Schwere der Geburt abhängig waren. Einige allgemeingültige Aussagen sollen an dieser Stelle aber getroffen werden. Beim Eintreten der ersten Wehen kamen neben der Hebamme - wenn es eine im Ort gab - auch weitere Frauen aus dem sozialen Umkreis der werdenden Mutter hinzu. Durchschnittlich waren während der Entbindung etwa fünf Frauen anwesend. Diese stopften alle Spalten der Türen und Fenster zu, um die Gebärende vor Kälte zu schützen. Zusätzlich sollten die werdende Mutter und ihr Nachwuchs durch die Abdichtung der Räumlichkeiten von schädlichen Einflüssen, wie etwa Schadenszaubern, beschützt werden. Generell wurde versucht, alles zu vermeiden, was der Frau Angst einflößen und dadurch die Niederkunft beeinflussen konnte. ${ }^{16}$ Die tatsächliche Entbindung fand auf einer Art Strohlager statt, das nach der Entbindung verbrannt wurde. Die Frauen brachten ihre Kinder normalerweise entweder im Stehen, Knien oder Sitzen zur Welt. Entband die Frau im Sitzen, wurde meist ein Gebärstuhl verwendet. Das Gebären im Liegen erfolgte nur dann, wenn die Frau erkrankte, zu erschöpft war oder es zu Komplikationen kam. Die anwesenden Frauen stützten währenddessen den Körper der Gebärenden oder führten andere assistierende Handlungen wie Wasserholen durch. In der letzten Phase der Geburt war es die Aufgabe der Hebamme, die Gebärende durch ihr Anpacken zu unterstützen und den Säugling aus dem Geburtskanal zu leiten. War das Kind auf der Welt, so wurde es der Mutter auf den Bauch gelegt, bevor die Nabelschnur durchtrennt wurde. AnschlieBend kümmerte sich die Hebamme um das Wohlergehen des Säuglings, während die restlichen Frauen die Mutter zu Bett brachten und sich um sie kümmerten. Schien das Kind schwächlich oder krank, musste eine Nottaufe und eine entsprechende Behandlung eingeleitet werden. Chirurgen und Bader wurden nur bei Knochenbrüchen oder Todesfällen hinzugerufen. War das Neugeborene gesund, wurde es in Tücher gewickelt und zur Mutter ins Bett gelegt. Die Praxis des sofortigen Säuberns und Wickelns des Kindes wurde im 18. Jahrhundert vorwiegend in Städten durchgeführt und setzte sich im ländlichen Raum erst im 19. Jahrhundert durch. Erst nach der erfolgten Niederkunft und den darauffolgenden Schritten wurde der Dienst der Hebamme und der anderen anwesenden Frauen als vollendet angesehen. ${ }^{17}$

Nach der Entbindung und den dazugehörigen Ritualen folgte die Zeit des Wochenbettes. Während dieser Phase befand sich die entbundene Frau in einer besonderen rituellen Position. Binnen dieser vier bis sechs Wochen standen der Mutter Frauen aus ihrem sozialen Umfeld durch Besuche und Hilfeleistungen bei. Die Entbundene war in

\footnotetext{
14 Marina Hilber, Institutionalisierte Geburt. Eine Mikrogeschichte des Gebärhauses, Bielefeld 2012, S. 45.

15 Horn, Wiener Hebammen, S. 85-94

16 Jacques Gélis, Die Geburt. Volksglaube, Rituale und Praktiken von 1500-1900, München 1989, S. 154-160.

17 Eva Labouvie, Andere Umstände. Eine Kulturgeschichte der Geburt, Köln 1998, S. $112-129$.
} 
dieser Zeit nämlich von der Gesellschaft isoliert. Sie war abgesondert von ihrem Ehegatten und durfte auch keine Gottesdienste besuchen. Die sogenannte "Aussegnung" der Wöchnerin durch einen Geistlichen beendete diese Phase und kann als Befreiung aus der Isolation und gleichzeitige Wiedereingliederung in die Gesellschaft gesehen werden. ${ }^{18}$

Die Säuglings- und Kindersterblichkeit sowie die Sterblichkeit der Gebärenden waren im 18. Jahrhundert hoch. Die statistischen Zahlen divergieren zwar von Region zu Region - sie waren unter anderem abhängig von medizinischen oder sozialen Umständen - durchschnittlich starben jedoch zwei bis drei von zehn Neugeborenen im ersten Lebensjahr, teilweise sogar fünf von zehn. ${ }^{19}$ Krankheiten, die bei Kindern oft zum Tod führten, waren unter anderem die Pocken, Scharlach, Röteln und fiebrige Erkrankungen. Trotz der hohen Sterblichkeit wurde aber weder der Tod des Kindes noch der Tod der Mutter "einberechnet" oder als Alltäglichkeit betrachtet. ${ }^{20}$ Bemerkte die Hebamme bei der Entbindung, dass das Neugeborene nicht überlebensfähig war, war die größte Furcht der Anwesenden, dass es nicht mehr rechtzeitig getauft werden konnte. Ungetaufte Kinder durften nicht auf Friedhöfen beigesetzt werden und laut damaligem Glauben war Ungetauften der Himmel verschlossen. Diese Furcht führte zu verschiedenen Praktiken, zum Beispiel dem Einführen von Weihwasser in die Gebärmutter. ${ }^{21}$ Zudem unterwies die katholische Kirche die Hebemütter beziehungsweise Hebammen, mit sterbenden Gebärenden zu beten, ihnen die Beichte abzunehmen sowie das Sakrament der (Not-)Taufe zu spenden. Eine weitere Aufgabe von Hebammen war, verstorbene Kinder und Mütter vor deren Beisetzung zu waschen und zu kleiden. ${ }^{22}$

\subsection{Bekanntgabe und Verlauf der Schwangerschaften Maria Theresias}

Nicht nur das Gebären brachte Herausforderungen mit sich, auch das frühe Erkennen einer Schwangerschaft war im 18. Jahrhundert eine Schwierigkeit. Das Ausbleiben der monatlichen Blutung konnte als Anzeichen einer Schwangerschaft wahrgenommen werden, allerdings konnte dies auch andere Gründe wie zum Beispiel Krankheiten haben. Medizinhistorischer Hintergrund war die im 18. Jahrhundert weiterhin geltende antike Viersäfte-Lehre beziehungsweise Humoralpathologie des Hippokrates und Galens. Laut dieser würde ein Ungleichgewicht der Körpersäfte Blut, Schleim, Schwarzer und Gelber Galle zu Erkrankungen führen. ${ }^{23}$ Eine Schwangerschaft galt als gesichert, wenn die Frau die ersten Kindsbewegungen spürte. Dies war meist im vierten oder fünften Monat der Fall. Maria Theresia bezeichnete in ihren Briefen den Nachwuchs ab dem Verspüren der Leibesbewegungen als „Kind“ (l'enfant). Zuvor verwendete sie die

18 Stollberg-Rilinger, Rituale, S. 61; Labouvie, Andere Umstände, S. 235-251.

19 Jürgen Schlumbohm, Kinderstuben. Wie Kinder zu Bauern, Bürgern, Aristokraten wurden, 1700-1850, München 1983, S. 25.

20 Labouvie, Andere Umstände, S. 158-167.

21 Stollberg-Rilinger, Rituale, S. 63.

22 Eva Labouvie, Beistand in Kindsnöten. Hebammen und weibliche Kultur auf dem Land (1550-1910) (Geschichte und Geschlechter 29), Frankfurt-New York 1999, S. 66-67.

23 Gélis, Die Geburt, S. 84-85. 
Bezeichnung „Frucht" (la fruit). ${ }^{24}$ Dass eine Schwangerschaft nicht so schnell als sicher erschien, lässt sich auch einem Brief Maria Theresias an ihre jüngste Tochter, Maria Antonia, entnehmen, in dem die Mutter die Königin von Frankreich bittet "zu beachten, daß zwei Monate nicht genug sind; dreizehn volle Wochen sind nötig, vor allem bei einer ersten Schwangerschaft, um Gewißheit zu haben"25.

Von einer Königin wurde erwartet, dass sie sowohl die Rolle der Ehefrau als auch der Mutter verkörperte. Indem sie Kinder gebar, sicherte sie den Fortbestand der Dynastie und deshalb war ihr Körper öffentlich. Auch der biologische Körper Maria Theresias lag daher im Interesse der Öffentlichkeit. ${ }^{26}$ Deshalb wurde eine Schwangerschaft, sobald sie durch die Kindsbewegungen gesichert war, der Öffentlichkeit verkündet. Unter anderem geschah dies dadurch, dass sich die Königin in einer prunkvollen Sänfte zur nächsten kirchlichen Feier tragen ließ. Dies galt als eindeutiges Zeichen für eine Schwangerschaft. Jener Akt wurde dann vom "Wienerischen Diarium" aufgenommen, abgedruckt und so einer noch breiteren Öffentlichkeit mitgeteilt. ${ }^{27}$ So geschah es zum Beispiel am 22. November 1740, als Maria Theresia "mit allen Ceremonien und Herzlichkeiten nach [...] St. Stephan [...] in einer Senften wegen Dero höchst-beglückten Schwangerschaft in schönster Ordnung getragen [...]" wurde.. ${ }^{28}$ Auch am 10. April 1748 wurde "Ihro Majest., die Kaiserin unsere Allergnädigste Landes-Fürstin wegen höchstbeglückten Schwangerschaft getragen [...]".29 Auch an andere Höfe wurde die Nachricht einer Schwangerschaft verschickt. ${ }^{30}$

Die Zeit der Schwangerschaft adeliger Frauen war von religiösen Bräuchen und Ritualen geprägt, in die auch die Untertanen miteinbezogen wurden. Nach der Verkündigung der Schwangerschaft einer Kaiserin sollten diese für die Herrscherin und deren Nachwuchs beten. Die Stände und der Klerus der habsburgischen Erblande wurden dazu angehalten, Andachten und Prozessionen für eine glückliche Schwangerschaft und Entbindung abzuhalten. ${ }^{31}$ Dafür wurden eigens Gebetstexte gedruckt, die an die Pfarreien geschickt wurden. ${ }^{32}$ In die Loretokapelle der Augustinerkirche in Wien spendeten die Habsburger*innen Opfergaben in Form von Votiven, um männlichen Nachwuchs zu erbeten. ${ }^{33}$ Um den Beistand der Gottesmutter zu erhalten, begab sich Maria Theresia während ihrer Schwangerschaften in eine Marienkapelle, entweder in Mariazell, Maria Hietzing oder die Loretokapelle, um dort zu beten. ${ }^{34}$

Für adelige Frauen war die Schwangerschaft der Normalzustand. Diese lagen meist auch sehr eng beieinander. Bei Maria Theresia betrug der zeitliche Abstand zwischen

24 Stollberg-Rilinger, Maria Theresia, S. 293.

25 Brief Maria Theresias an Marie Antoinette, 2.5.1778, zit. nach Paul Christoph (Hrsg.), Maria Theresia und Marie Antoinette. Der geheime Briefwechsel, Darmstadt 2017, S. 253.

26 Banakas, Die zwei Körper, S. 78.

27 Stollberg-Rilinger, Maria Theresia, S. 292.

28 Wienerisches Diarium, 23.11.1740, S. 6.

29 Wienerisches Diarium, 13.4.1748, S. 6.

30 Stollberg-Rilinger, Maria Theresia, S. 292.

31 Sabine Weiss, Die Österreicherin. Die Rolle der Frau in 1000 Jahren Geschichte, Graz-Wien-Köln 1996, S. 15-16.

32 Stollberg-Rilinger, Maria Theresia, S. 298.

33 Vocelka/Heller, Die private Welt, S. 27.

34 Stollberg-Rilinger, Maria Theresia, S. 298 
zwei Entbindungen höchstens zwei Jahre. Die adeligen Damen waren aber privilegiert, denn im Unterschied zu anderen Gesellschaftsschichten mussten sie während der Schwangerschaft nicht körperlich arbeiten. Zusätzlich waren sie dadurch bevorteilt, dass ihnen jeweils die beste medizinische Hilfe ihrer Zeit zugesichert war. An den Folgen von mehreren Schwangerschaften starben allerdings etliche adelige Frauen, denn oft war der Körper der jung Verheirateten nicht bereit für die hohe Anzahl an Schwangerschaften. Es ist zudem anzumerken, dass über eine genaue Kinderanzahl beziehungsweise Anzahl der Schwangerschaften oft keine sichere Aussage gemacht werden kann, da Fehlgeburten oft nicht vermerkt wurden. Dies lag daran, dass die ungetauften Nachkommen nicht in den Taufbüchern aufschienen, welche die Hauptquelle für Bevölkerungsstatistiken aus dieser Zeit darstellen. ${ }^{35}$

Während der Schwangerschaft wurden an der Herrscherin drei Aderlässe durchgeführt, um deren Wohlbefinden zu bessern. ${ }^{36}$ Gemäß der Viersäfte-Lehre galt, dass während der Menstruation Schadstoffe aus dem Blut gefiltert worden wären. Während der Schwangerschaft sollte das Ausbleiben der monatlichen Blutung also mit einem Aderlass kompensiert werden. An Maria Theresia wurde der Aderlass meist je einmal zu Beginn, in der Mitte und am Ende der Schwangerschaft durchgeführt. Dieser hatte nicht nur eine medizinische Komponente, sondern war gleichzeitig ein soziales Ereignis, das in Anwesenheit der Hofgesellschaft stattfand und im „Wienerischen Diarium” publik gemacht wurde. Der Hofstaat fand sich in festlicher Kleidung bei der Königin ein, gratulierte ihr zum Aderlass und widmete sich unterschiedlichen Unterhaltungen. Besonders seit Maria Theresia die Herrschaft von ihrem Vater übernommen hatte, wurde der Aderlass als höfischer Galatag angesetzt. ${ }^{37}$ So wurde sie auch im April 1747 kurz vor der Entbindung Leopolds zur Ader gelassen, wozu der hohe Adel erschien, um Gratulationen zu überbringen. ${ }^{38}$ Am 19. Juli 1752 wurde nach dem letzten Aderlass ein "großes Spiel" abgehalten. ${ }^{39}$

In den Monaten vor der Entbindung wurde das Personal erwählt, welches die Kaiserin und ihr Kind während und nach der Geburt umsorgen sollte. ${ }^{40}$ Auswahlkriterien für Ammen waren beispielsweise deren Kinderanzahl, Manieren sowie Lebensstil. ${ }^{41}$ Sie sollte zwischen 18 und 20 Jahre alt sein und selbst vor sechs Wochen entbunden haben. Im Idealfall hatte sie bereits mindestens zwei Kinder, damit man sich von deren Wachstum ein Bild machen konnte. Außerdem wurde auf die Brust, Milch, Haarfarbe und das Temperament einer jeweiligen Amme geachtet. Maria Theresia ließ ihre Ammen zuerst von ihrem Leibarzt begutachten und veranlasste ihre Verpflegung. ${ }^{42}$ Zusätzlich zu den Personalbestimmungen wurde kurz vor der Niederkunft eine Hofkonferenz einberufen, in der allfällige Eventualitäten und zeremonielle Fragen rund um die Entbindung behan-

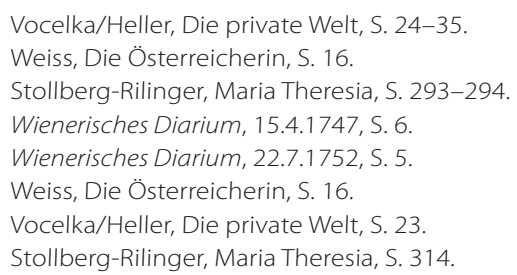


delt wurden. Eine derartige Konferenz wurde etwa 1741 einberufen, als Maria Theresia mit ihrem vierten Kind schwanger war. Darin wurde der Vorgang besprochen, wie mit der erneuten Geburt eines Mädchens umgegangen werden sollte und inwiefern die Feierlichkeiten in Anbetracht der Hoftrauer des kurz zuvor verschiedenen Vaters Maria Theresias, Kaiser Karl VI., gestaltet werden sollten. Die Geburt des Thronfolgers Josephs war mit großer Freude verbunden, sodass Maria Theresia trotz der Trauer das übliche, üppige Zeremoniell abhalten ließ.43

\subsection{Maria Theresias Idealbild einer Schwangerschaft}

Das Zeremoniell bestimmte auch die Schwangerschaft. Die schwangere Fürstin musste sich gewissen Verhaltensregeln unterwerfen. Sie sollte sich während dieser Zeit schonen. Sowohl für die Schwangere als auch für das ungeborene Kind wurden überall Gefahren befürchtet. Mit dieser Begründung durfte die schwangere Maria Theresia im Oktober 1740 ihren Vater, Kaiser Karl VI., nicht an dessen Sterbebett besuchen, denn der schreckliche Anblick konnte nach zeitgenössischer Auffassung die Gesundheit des Kindes gefährden. Aus demselben Grund wurden Bettler*innen vom Hofgelände verbannt. Oft wurden auch die Fenster mit Stoffen zugehängt, damit Schwangere vor Anblicken geschützt waren, die dem Ungeborenen womöglich schadeten. Zudem sollte weder geritten, getanzt noch über holprige Straßen gefahren werden.

Maria Theresia ertrug ihre Schwangerschaften und Entbindungen, gerade bei den ersten Kindern, im Großen und Ganzen mühelos. So soll sie nach der Entbindung Josephs den Wunsch geäußert haben, möglichst bald wieder schwanger zu sein. Die Königin ließ sich durch die Schwangerschaften weder in ihrem streng geplanten Tagesrhythmus noch in ihrem Lebenswandel einschränken. So betrieb sie weiterhin die oben genannten Aktivitäten und wurde deshalb von ihrem Umfeld kritisiert. Dies änderte sich allerdings, nachdem ihre Schwester Maria Anna im Kindbett ums Leben kam und sie selbst 1748 mit Maria Karolina ein Kind zur Welt brachte, das kurz nach der Entbindung verstarb. Maria Theresia glaubte, dass eine zuvor unternommene Kutschenfahrt der Auslöser dafür gewesen sei. Auch ihren Töchtern sowie ihren Schwiegertöchtern legte sie später nahe, sich an die vorgegebenen Gebote zu halten - im Gegensatz zu ihrer eigenen Person. ${ }^{44}$

Ihrer Schwiegertochter Maria Beatrice ${ }^{45}$ (1750-1829), die sich ihrer Schwangerschaft noch nicht sicher war, riet sie, sich zu schonen „selbst wenn es umsonst wäre, man kann nicht vorsichtig genug sein. [...] Spätes Heimkommen abends, das kann ich nicht gutheißen".46 Weiters sollte sie "solange es noch keine Gewißheit gibt, von allen an-

43 Irene Kubiska, Und ist wegen dieser so glücklich- und trostreichen Geburth ein allgemeines Frolockhen und grosse Freydt gewesen. Das Geburten- und Taufzeremoniell am Wiener Hof im Zeitraum von 1652 bis 1800, in: Irmgard Pangerl/Martin Scheutz/Thomas Winkelbauer (Hrsg.), Der Wiener Hof im Spiegel der Zeremonialprotokolle (1652-1800). Eine Annäherung (Forschungen zur Landeskunde von Niederösterreich 31, Forschungen und Beiträge zur Wiener Stadtgeschichte 47), Innsbruck-Wien 2007, S. 493-527, hier S. 503-505.

44 Stollberg-Rilinger, Maria Theresia, S. 294-295.

45 Ehefrau von Ferdinand Karl. Das Paar gilt als Begründer des Hauses Österreich-Este.

46 Brief Maria Theresias an Maria Beatrice, 28.5.1772, zit. nach Rothe, Die Mutter und die Kaiserin, S. 333. 
strengenden langen Wagenfahrten und Spaziergängen" absehen. ${ }^{47}$ Maria Theresia war sich auch bewusst, wie kritisch die ersten Monate einer Schwangerschaft sind. Sie äußerte, dass „Aufregungen jeglicher Art [...] gleichermaßen für das Herz sehr schädlich [...] und am meisten in den ersten drei Monaten" seien. ${ }^{48}$ Ihren Sohn Ferdinand bat sie, nachdem dessen einjähriger Sohn gestorben war: „[S]agt Eurer Gemahlin nichts von dem, was man bei der Öffnung der Leiche des Kindes festgestellt hat, so etwas taugt nicht für eine Frau, die ein Kind erwartet."49

Im Briefverkehr mit ihrer jüngsten Tochter, Maria Antonia, die 1770 mit dem Dauphin von Frankreich verheiratet wurde, war eine Schwangerschaft aus den bereits bekannten Gründen das zentrale Thema. Nach jahrelangem Warten teilte die Königin von Frankreich ihrer Mutter mit, dass sie glaubte, schwanger zu sein. Vielleicht war Maria Theresia gerade aufgrund des langen Wartens auf eine derartige Nachricht besonders besorgt um die Schwangerschaft ihrer Tochter, als sie sie wegen des bevorstehenden Karnevals warnte. Es wäre „unverzeihlich, wenn Du [...] zu solcher Jahreszeit Dich der Gefahr aussetzen [...] würdest. Deine Gesundheit [...] gehört nicht Dir allein". ${ }^{0}$ Auch die Königin von Frankreich teilte ihren Körper nicht nur mit ihrem ungeborenen Kind, sondern er war ebenso der Körper der Öffentlichkeit und des Staates, wie es auch schon bei ihrer Mutter der Fall gewesen war. Nach einer enttäuschten Schwangerschaftsvermutung oder einer möglichen Fehlgeburt Maria Antonias sollte sie laut ihrer Mutter „selbst die Schuld daran haben, weil sie in der Zeit der ersten Schwangerschaftsanzeichen getanzt haben soll'.51

Am wenigsten ließ sich Maria Theresia einschränken, wenn es um Regierungsgeschäfte ging. So führte sie diese bis kurz vor einer Entbindung fort und einige Tage nach dieser kehrte sie zu ihnen zurück. ${ }^{22}$ Diese Haltung gegenüber der Pflichterfüllung wusste sie auch geschickt gegen ihre Minister einzusetzen. Am 19. Februar 1745, also 18 Tage nach der Geburt von Karl Joseph, kritisierte sie: „Mein Kindbett ist aus, sie aber haben lange Vacanzen [...]"53. Auch Khevenhüller-Metsch kommentierte, dass die Königin „ob sie schon noch nicht vorgeseegnet, dennoch bereits mit den Ministris widerummen zu arbeiten angefangen" ${ }^{54}$. Die Aussegnung diente als Reinigungszeremonie nach der Geburt und die Wöchnerin konnte eigentlich nur durch dieses Ritual wieder ihren Platz in der Gesellschaft einnehmen. ${ }^{55}$ Neben den politischen Tagesgeschäften nahm Maria

47 Brief Maria Theresias an Maria Beatrice, 28.5.1772, zit. nach Rothe, Die Mutter der Kaiserin, S. 334

48 Brief Maria Theresias an Maria Beatrice, 8.3.1773, zit. nach ebd., S. 336.

49 Brief Maria Theresias an Sohn Ferdinand, 26.8.1776, zit. nach ebd., S. 315.

50 Brief Maria Theresias an Marie Antoinette, 5.1.1778, zit. nach Walter, Maria Theresia, S. 414.

51 Brief Maria Theresias an Graf Mercy d'Argenteau, Wiens Gesandter in Paris, 31.7.1779, zit. nach Rothe, Die Mutter und die Kaiserin, S. 402.

52 Hier sei allerdings anzumerken, dass sich Österreich während der meisten Geburten Maria Theresias im Krieg mit Preußen befand, was dahingehend ein Faktor gewesen sein dürfte: Stollberg-Rilinger, Maria Theresia, S. 295.

53 Brief Maria Theresias an den Hofkammerpräsidenten Johann Franz Gottfried Graf Dietrichstein, 19.2.1745, zit. nach Rothe, Die Mutter und die Kaiserin, S. 35.

54 Tagebuch des kaiserlichen Obersthofmeister Johann Josef Khevenhüller-Metsch, 1.9.1743, zit. nach Rudolf Khevenhüller-Metsch/Hanns Schlitter (Hrsg.), Aus der Zeit Maria Theresias. Tagebuch des Fürsten Johann Josef Khevenhüller-Metsch. Kaiserlichen Obersthofmeisters. 1742-1776, Bd. 1, Leipzig-Wien 1907, S. 174.

55 Eva Labouvie, Selbstverwaltete Geburt. Landhebammen zwischen Macht und Reglementierung (17.-19. Jahrhundert), in: Geschichte und Gesellschaft 18 (1992), Heft 4, S. 447-506, hier S. 502-503. 
Theresia auch die religiösen Verpflichtungen weiterhin wie gewohnt wahr, nur vom Fastengebot ließ sie sich ausnehmen. In der letzten Phase der Schwangerschaften nahm sie nicht mehr an langen Prozessionen oder an manchen Zusammenkünften des engeren Hofstaates teil, ansonsten ließ sich die Königin nicht besonders einschränken. ${ }^{56}$

Im Laufe der Zeit nahm Maria Theresia die Schwangerschaften vermehrt als Einschränkung ihres Bewegungsspielraumes, ihrer Arbeitskraft und Gemütslage wahr. Mit zunehmendem Alter scheint Maria Theresia ihre Schwangerschaften als Last empfunden zu haben. So schrieb sie an ihre enge Vertraute Maria Antonia von Sachsen kurz vor der Geburt des zehnten Kindes, dass sie sich keine weiteren Kinder mehr wünschte, "denn ich fühle, daß es mich schwächt und recht altern läßt und für alle Kopfarbeit weniger fähig macht ${ }^{\prime 57}$. Es sollten aber noch sechs weitere Kinder folgen. Kurz nach jener zehnten Entbindung schrieb Maria Theresia, dass sie befürchtete, wieder schwanger zu sein. Dies sollte sich auch als richtig herausstellen, denn im Februar 1750 kam eine weitere Tochter zur Welt. ${ }^{58}$ Im Oktober 1761 zeigte sie sich in einem weiteren Brief an Maria Antonia von Sachsen erfreut "zu vernehmen, daß Sie nicht schwanger sind [... ]"59. Diese Briefpassage spiegelte wohl die zunehmend negative Einstellung Maria Theresias gegenüber weiteren Schwangerschaften wider. Obwohl viele Entbindungen dem Erhalt der Dynastie dienten und die Hauptaufgabe der Frauen waren, sprach sie Glückwünsche zu einer nicht eingetretenen Schwangerschaft ihrer Freundin aus. Über die Entbindung schrieb sie in einem Brief 1744 an ihre schwangere Schwester: „Ich

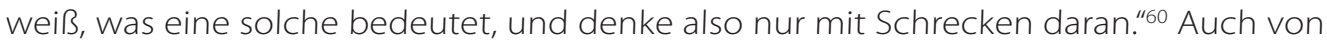
anderen wurden die Auswirkungen der Schwangerschaften auf den Körper der Kaiserin bemerkt. Der preußische Gesandte schrieb 1747, dass ihre Figur "vor ihrer Heirat sehr schön [war], aber die zahlreichen Geburten, die sie durchgemacht hat, [...] haben sie äußerst schwerfällig werden lassen"61.

\section{Die Entbindungen Maria Theresias}

Die Geburten am Herrscherhof und die damit verbundenen Rituale waren durch das Zeremoniell, das unmittelbar mit der Herrschaft verbunden war, geregelt. ${ }^{62}$ Es demonstrierte den Rang, die Zugehörigkeit und Funktion mithilfe von Ritualen und bestätigte immer wieder das gesellschaftliche Gefüge.63 Dies führte dazu, dass beispielsweise jeder Schritt, jede Geste, die Bekleidung sowie die Etikette fest geregelt und vorgegeben

56 Stollberg-Rilinger, Maria Theresia, S. 295.

57 Brief Maria Theresias an Maria Antonia Kurprinzessin von Sachsen, O. D. [ca. August 1748], zit. nach Friedrich Walter (Hrsg.), Maria Theresia. Briefe und Aktenstücke in Auswahl (Ausgewählte Quellen zur Deutschen Geschichte der Neuzeit 12), Darmstadt 1968, S. 58.

58 Walter, Maria Theresia, S. 62.

59 Brief Maria Theresias an Maria Antonia Kurprinzessin von Sachsen, 6.10.1761, zit. nach ebd., S. 160.

60 Brief Maria Theresias an Schwester Marianne, 3.10.1744, zit. nach ebd., S. 39.

61 Otto Christoph Graf Podewils an Friedrich II., 18.1.1747, zit. nach Wolf H. Birkenbihl, Maria Theresia - Monarchin, Mutter und Mensch. Ihr Leben und ihre Zeit in Briefen und Augenzeugenberichten, Baden-Baden $2017,5.58$.

62 Vocelka/Heller, Die Lebenswelt, S. 249

63 Hubert Ch. Ehalt, Ausdrucksformen absolutistischer Herrschaft. Der Wiener Hof im 17. und 18. Jahrhundert (Sozial- und Wirtschaftshistorische Studien 14), Wien 1980, S. 114-116. 
waren. ${ }^{64}$ Im Folgenden werden Ort(e) der Entbindungen und die dabei Anwesenden thematisiert.

\subsection{Ort(e) der Entbindungen}

Neben deren gesellschaftlichem Stellenwert sind Rituale fast immer an einen spezifischen Ort gebunden, der den Übergang von Alltag zu Festtag konkretisiert. ${ }^{65}$ Bei einem Ortswechsel wurde das Hofzeremoniell an die jeweiligen Örtlichkeiten angepasst. Maria Theresia bewohnte mit ihrem Hofstaat den größten Teil des Jahres die Wiener Hofburg. Während der Sommermonate - meist von April bis Oktober - übersiedelte der Hof nach Schloss Schönbrunn. Abhängig war die Dauer des Aufenthaltes von der Witterungslage, aber auch vom liturgischen Kalender. ${ }^{66}$

Die Entbindungen Maria Theresias fanden ebenso wie Taufen und Beerdigungen prinzipiell in der Wiener Hofburg statt, nur in Ausnahmefällen wurden sie in andere Residenzen verlegt. Die ersten acht Kinder entband die Königin in der Hofburg, danach beschloss sie, in Schönbrunn zu entbinden, wenn die Niederkunft in die Sommermonate fiel. ${ }^{67}$ So erblickten Leopold (1747), Maria Karolina (1748), Maria Karolina (1752) und Ferdinand Karl (1754) im Schloss Schönbrunn das Licht der Welt. Wenn Maria Theresia in der Hofburg gebar, dann tat sie dies auf der Frauenseite ihrer Appartements. Hielt sich die Königin in Schönbrunn auf, so mussten die Tore der Residenzstadt geöffnet bleiben, damit im Falle der Geburt schnell in die Hofburg zurückgekehrt werden konnte. Dies betraf jene Geburten, die in die Sommermonate vor dem Beschluss des Gebärens in Schönbrunn fielen. Wenn sie aber in Schönbrunn gebar, so mussten die hohen Positionen der Hofgesellschaft zu der Entbindung hinzukommen. ${ }^{68}$ Dass die Übersiedelung der Gebärenden von Schönbrunn in die Hofburg nicht immer leicht war, zeigte die Geburt von Erzherzogin Maria Elisabeth am 13. August 1743. Dabei musste Maria Theresia in der Hofburg einige Zeit warten, bis ihr das Gebärzimmer geöffnet wurde:

„[...] den 13. gegen anbrechenden Tag [...] fiengen dieselbe an einige Vorbotten herannahender Geburt zu spühren, weßwegen sie sich [...] in die Purg herein begaben; und obschon I. M. [Ihre Majestät] würklich im Fahren unterweegs eine und andere sogenannte schleichende Wehe empfanden und noch über dises aus eine Fatalitet - weilen der Zimmerwarter, der sich darauf nicht versehen, mithin auch sogleich nicht an der Hand gewesen, um dero Wohnungs Zimmern aufzuspören - gegen einer Viertlstund in der Trabantenstuben warten musten, gienge doch alles Gottlob ganz glücklich von statten [...]."69

64 Vocelka/Heller, Die Lebenswelt, S. 249.

65 Nicolas Pethes, Kulturwissenschaftliche Gedächtnistheorien zur Einführung, Hamburg 2008, S. 87.

66 Marina Beck, Macht-Räume Maria Theresias. Funktion und Zeremoniell in ihren Residenzen, Jagd- und Lustschlössern (Kunstwissenschaftliche Studien 189), Berlin-München 2017, S. 147.

67 Ebd., S. 84

68 Stollberg-Rilinger, Maria Theresia, S. 299.

69 Khevenhüller-Metsch/Schlitter, Tagebuch des Fürsten Khevenhüller-Metsch, Bd. 1, S. 172. 


\subsection{Die Anwesenden}

Auch am Kaiserhof waren Entbindungen Frauensache. Sobald sich die Kaiserin mit Wehen in ihr Schlafgemach zurückzog, versammelten sich die Frauen des Hofes um sie. Sie gaben der werdenden Mutter Ratschläge, die im Gebärstuhl entband. Gleichzeitig kontrollierten sie auch die Hebamme, die bei Komplikationen meist verantwortlich gemacht wurde..$^{70}$ Die Niederkunft einer Herrscherin war sowohl ein soziales als auch ein politisches Ereignis. Daher waren neben den Familienangehörigen auch die Spitzen des Hofes vertreten - wohlgemerkt nur die weiblichen. Die männlichen Angehörigen mussten in der Ritterstube auf das Neugeborene warten. ${ }^{71}$ Diese für alle Habsburgerinnen typische Konstellation sollte sich mit der Berufung Gerard van Swietens (17001772) als Leibarzt Maria Theresias nach und nach ändern.

Gerard van Swieten studierte als Schüler des Arztes Hermann Boerhaave in Leiden. ${ }^{72}$ Als dieser 1727 erkrankte, übernahm van Swieten interimistisch dessen Professur. Nach dem Ableben des Mentors sollte van Swieten dieses Amt dauerhaft übernehmen, was inm jedoch als Katholik im protestantischen Leiden verwehrt wurde. ${ }^{73} 1743$ erhielt er eine Einladung an den Wiener Hof, die er zunächst ablehnte. ${ }^{74} 1744$ wurde er an das Wochenbett der Schwester Maria Theresias, Maria Anna, nach Brüssel gerufen. ${ }^{75} \mathrm{Ob-}$ wohl er ihr nicht mehr helfen konnte und Maria Anna verstarb, war Maria Theresia doch von seinem Können überzeugt und rief ihn 1745 als ihren Leibarzt und Hofbibliothekar an den Wiener Hof, was er diesmal annahm. ${ }^{76}$ Mit van Swieten erreichte Wien auch den Anschluss an die moderne Medizin. Er vollzog eine Universitätsreform und gründete die erste Wiener Medizinische Schule. ${ }^{77}$ Van Swieten bemühte sich zudem um eine verbesserte Ausbildung der Hebammen. ${ }^{78}$ So führte er 1754 den ersten Lehrstuhl für Hebammenkunst ein und reglementierte daraufhin die Hebammenausbildung. ${ }^{79}$ Maria Theresia vertraute ihm zudem ihre Kinder an und deren Erzieher*innen waren verpflichtet, van Swieten genauestens über den Gesundheitszustand der Kinder zu berichten. Auch sich selbst übergab die Kaiserin ganz in die Obhut eines einzigen Arztes. Für Fürstenhäuser jener Zeit war es sonst üblich mehrere Ärzte zu haben. ${ }^{80}$

70 Sabine Weiss, Zur Herrschaft geboren. Kindheit und Jugend im Haus Habsburg von Kaiser Maximilian I. bis Kronprinz Rudolf, Innsbruck 2008, S. 35.

71 Stollberg-Rilinger, Maria Theresia, S. 299.

72 Heinz Huber, Pioniere aus Medizin- und Geschichtswissenschaften in Österreich. Ausgewählte Biographien aus über zwei Jahrhunderten, Innsbruck 2017, S. 3.

73 Werner E. Gerabek, Swieten, Gerard van (Gerhard von), in: Neue Deutsche Biographie, Bd. 25, Berlin 2013, S. 729 730.

74 Hanne Egghardt, Maria Theresias Männer. Ihre Lieben, ihre Ratgeber und die Stützen ihres Throns, Wien 2015, S. 111.

75 Vocelka/Heller, Die private Welt, S. 170.

76 Huber, Pioniere, S. 3.

77 Vocelka/Heller, Die private Welt, S. 170.

78 Huber, Pioniere, S. 3.

79 Stollberg-Rilinger, Maria Theresia, S. 301.

80 Monika Gertraud Gasser, Das Gesundheitsverhalten von Herrscherinnen und Herrschern des 18. Jahrhunderts im Vergleich. Dargestellt am Beispiel von Maria Theresia, Friedrich dem Großen, Marie Antoinette und Joseph II., Dipl. Innsbruck 2005, S. 18-19. 
Mit der Ankunft van Swietens in Wien änderte sich auch die Art und Weise, wie Maria Theresia ihre Kinder gebar. Hatte sie ihre ersten sechs Kinder noch in gewohnter Weise - im Gebärstuhl, umgeben von Frauen - zur Welt gebracht, entband sie die restlichen zehn im Bett liegend und in Anwesenheit von lediglich einer Hebamme und ihrem Leibarzt. ${ }^{81}$ Sobald sich der Termin der Niederkunft näherte, bezog die ausgewählte Hebamme einen Raum am Aufenthaltsort der Kaiserin. Da die Anwesenheit bei den Entbindungen der Herrscherin als Privileg wahrgenommen wurde, erwarteten die Frauen der Hofgesellschaft eine symbolische Kompensation dafür, dass sie nun dem Entbindungszimmer fernbleiben mussten, um vor der Hofgesellschaft nicht das Gesicht zu verlieren. Deshalb wurden die Hofdamen früher zum Handkuss vorgelassen, als es bis dahin üblich gewesen war. Unter van Swieten kam es auch zu einer Verdrängung der Religion aus dem Entbindungszimmer zugunsten der Wissenschaft. So veranlasste er beispielsweise, dass dort keine Reliquien mehr aufgestellt wurden. ${ }^{82}$

Wie überzeugt Maria Theresia von dem Einzug der Ärzte im Gebärzimmer war, kam in einem Brief an die schwangere Tochter Maria Antonia zum Ausdruck, indem sie hoffte, dass ihr Leibarzt den Geburtshelfer auswählte und "daß es ein erfahrener und christlicher Mann ist. [...] Ich bin immer sehr gut dabei gefahren, jenen die Wahl zu überlassen, die am besten ihr Wissen anzuwenden verstehen"83. Auffällig ist, dass van Swieten und die Hebamme in den Aufzeichnungen über die Entbindungen als Akteur*innen kaum auszumachen sind. Namentlich wird die Hebamme beispielsweise in Khevenhüller-Metschs Tagebüchern überhaupt nie erwähnt.

\subsection{Rituale und Ablauf der Entbindungen}

Da die Herrschaft innerhalb der Familie weitergegeben wurde, fiel lebenszyklischen Ritualen wie Geburt, Taufe, Hochzeit und Tod eine politische Bedeutung zu. Sie dienten der Familie dazu, deren reale, soziale sowie kulturelle Macht vor den Untertanen, dem Adel und auch konkurrierenden Herrscherfamilien zu demonstrieren, was natürlich besonders bei männlichem Nachwuchs funktionierte. ${ }^{84}$ In die Rituale um die Geburt flossen auch religiöse Elemente ein, die in der Frömmigkeit der Habsburger*innen, der sogenannten pietas Austriaca, begründet waren. ${ }^{85}$

Als sich abzeichnete, dass die Niederkunft der Herrscherin nicht mehr weit entfernt war, wurden die bereits erwähnten Gebete für die Königin und deren Nachwuchs verstärkt. In der Kammerkapelle des jeweiligen Schlosses wurde das Allerheiligste ausgesetzt und in der Residenzstadt Wien wurde eine Art Gebetskette der Untertanen in verschiedenen Kirchen gebildet. Auch nachts wurde am Kaiserhof durchgehend für die Königin gebetet. ${ }^{86}$ Als die Wehen einsetzten, zog sich die Kaiserin in ihr Schlafgemach zurück.

81 Weiss, Zur Herrschaft geboren, S. 35.

82 Stollberg-Rilinger, Maria Theresia, S. 300-303.

83 Brief Maria Theresias an Marie Antoinette, 2.5.1778, zit. nach Christoph, Maria Theresia und Marie Antoinette, S. 253.

84 Stollberg-Rilinger, Rituale, S. 56-57.

85 Vocelka/Heller, Die Lebenswelt, S. 274

86 Stollberg-Rilinger, Maria Theresia, S. 298-299. 
Dorthin wurden dann auch die Reliquien gebracht. ${ }^{87}$ Derweil versammelte sich die kaiserliche Familie zum Gebet in der Kapelle. ${ }^{88}$ In den Zeremonialprotokollen erschienen die Entbindungen oft als nüchterner Sachvermerk. Bezüglich der Geburt wurde nur vermerkt, ob sie erfolgreich verlaufen war oder nicht. Auch Datum und Uhrzeit wurden dokumentiert. Der Gesundheitszustand des neugeborenen Kindes und der Entbundenen wurden kaum erwähnt. ${ }^{89}$ So vermerkte Oberst Khevenhüller-Metsch im Februar des Jahres 1746 in seinem Tagebuch „den 26. gegen 11 Uhr nachts wurden I.M. [Ihre Majestät] die Kaiserin einer Erzherzogin [Maria Amalia] gantz glücklich entbunden".90

Am 12. März 1741 gegen 23 Uhr setzten bei Maria Theresia die Wehen bei ihrer vierten Entbindung ein und etwa eine Stunde später wurde das Allerheiligste in der Kammerkapelle in der Augustinerkirche ausgesetzt. Obersthofmeisterin Gräfin Fuchs und die obersten Hofdamen versammelten sich im Gebärzimmer der Königin. Maria Theresia wurde am darauffolgenden Tag kurz nach zwei Uhr vom Thronfolger Joseph entbunden. ${ }^{91}$ Wie bedeutend die Geburt Josephs war, spiegelt sich auch in einem Brief Maria Theresias an eine ihrer engsten Vertrauten, die Gräfin Edling, vom 12. März 1766 wider. Darin schrieb sie: „Heute vor fünfundzwanzig Jahren um zwei Uhr in der Nacht war der glücklichste Tag, den ich in meinem Leben gehabt, nach dem 12. Februar 1736 [Hochzeitstag]. ${ }^{192}$ Diesem Schema folgend betonte sie in einem Schreiben im März 1777 an ihren Sohn Ferdinand die Wichtigkeit der Geburt Josephs, insbesondere auf den Erbfolgekrieg bezogen. Es lässt sich an dieser Stelle gut erkennen, welche Bedeutung dieses männliche Kind hatte:

„Welch ein großer Tag für mich, der all meine Handlungen von vor sechsunddreißig Jahren wieder lebendig macht und der mir auch die Hoffnung gegeben hat, daß der liebe Gott, diese göttliche Vorsehung, das Szepter unserem Hause belassen wollte, weil er mir im kritischsten Augenblicke einen Sohn gewährte. [...] ich hatte kein Königreich mehr, [...] so daß ich [...] nicht einmal mehr wußte, wo ich niederkommen sollte [...]."93

Der zweite Sohn Maria Theresias und Franz Stephans wurde am 1. Februar 1745 geboren. Über das Geburtsgeschehen selbst vermerkte Khevenhüller-Metsch, dass die Königin glücklich entbunden wurde und was Franz Stephan den Versammelten im Spiegelsaal verkündete. Auch der zweite Sohn, Karl Joseph, wurde mit großer Freude empfangen, wie aus folgendem Eintrag Khevenhüller-Metschs hervorgeht:

87 Zumindest bis zur Zeit van Swietens, wie vorhin erwähnt.

88 Weiss, Zur Herrschaft geboren. S. 35.

89 Kubiska, Geburten- und Taufzeremoniell am Wiener Hof, S. 495.

90 Khevenhüller-Metsch/Schlitter (Hrsg.), Aus der Zeit Maria Theresias, Bd. 2, S. 78-79, zit. nach Birkenbihl, Maria Theresia, S. 56.

91 Weiss, Zur Herrschaft geboren, S. 248-249.

92 Brief Maria Theresias an Gräfin Rosalie von Edling, 12.3.1766, zit. nach Maria Theresia, Familienbriefe. Mit einem biographischen Anhang (Die Fünfzig Bücher 3), Berlin-Wien 1916, S. 108.

93 Brief Maria Theresias an Erzherzog Ferdinand, 13.3.1777, zit. nach Walter, Maria Theresia, S. 403. 
„Bald darauf kamme auch die Kaiserin aus der Cammer heraus und ob sie zwar ihres bekanten Zustands halber so schwach an Füssen, daß sie kaum stehen kann und sich immer tragen lassen muß, gieng sie aus der nemmlichen Emotion von Freuden mit verwunderlicher Geschwindigkeit zum Fenster, um solches zu eröffnen und selbsten hinunter zu schreien, daß ein Ertzherzog gebohren worden seie.".94

Am 17. September 1748 wurde die Herrscherin von einer Tochter entbunden. Laut dem entsprechenden Tagebucheintrag von Khevenhüller-Metsch hatte Maria Theresia gegen Mittag die ersten Wehen verspürt. Daraufhin wurde sie zur Ader gelassen, was ohnehin für den nächsten Tag angesetzt gewesen war. Es wurde vermutet, dass die Entbindung noch einige Stunden entfernt lag, weshalb sich Kaiser Franz mit einigen Gefolgsleuten zum Spaziergang in den Garten begab. Kurz darauf wurden sie zurückgerufen, wo es bald hieß, , die Kaiserin wäre würklichen entbunden; jedoch wollte niemand sagen, wie es mit selber stünde und ob es ein Sohn oder eine Dochter seie"95. Als Frauen aus dem Gebärzimmer kamen, herrschte große Bestürzung und es wurde befürchtet „üble Nachricht zu vernehmen"96. Schließlich wurde bekannt, dass das Kind tot war. $^{97}$

Nach den Entbindungen war es üblich, dass ausgewählte Personen zum Handkuss an das Wochenbett gelassen wurden. Dies geschah nur, wenn dies der Gesundheitszustand der Mutter erlaubte und fand meistens einige Zeit nach der Entbindung statt. Bei den ausgewählten Personen handelte es sich um den päpstlichen Nuntius, Botschafter und Stadtdamen. ${ }^{98}$ Noch im selben Monat der Geburt Maria Christines im Mai 1742 ließ die Königin Kammerfrauen und Hofdamen in ihr Schlafzimmer eintreten und erlaubte ihnen, ihr die Hand zu küssen. Bei Karl Joseph geschah dies zehn Tage nach der Geburt. Maria Theresia war zu diesem Zeitpunkt bereits aufgestanden. Bei den Geburten von Maria Josepha und Maria Antonia verlief es ähnlich. ${ }^{99}$

Als Maria Beatrice d'Este von ihrer Schwiegermutter Maria Theresia Ratschläge zu einer ihrer bevorstehenden Entbindungen erbat, schickte die Königin eine Hebamme in die Lombardei. ${ }^{100}$ In einem begleitenden Brief gestand sie ihrer Schwiegertochter „ich kann Euch nicht einmal einen ernsthaften Rat geben, denn trotz meiner sechzehn Kinder weiß ich nicht, rein gar nichts"101. Das Unwissen war ihr zufolge beabsichtigt: „was

94 Tagebuch des kaiserlichen Obersthofmeister Johann Josef Khevenhüller-Metsch, 31.1.1745, zit. nach Rudolf Khevenhüller-Metsch/Hanns Schlitter (Hrsg.), Aus der Zeit Maria Theresias. Tagebuch des Fürsten Johann Josef Khevenhüller-Metsch. Kaiserlichen Obersthofmeisters. 1742-1776, Bd. 2, Leipzig-Wien 1908, S. 22.

95 Tagebuch des kaiserlichen Obersthofmeister Johann Josef Khevenhüller-Metsch, 17.9.1748, zit. nach KhevenhüllerMetsch/Schlitter, Tagebuch des Fürsten Khevenhüller-Metsch, Bd. 2, S. 262

96 Tagebuch des kaiserlichen Obersthofmeister Johann Josef Khevenhüller-Metsch, 17.9.1748, zit. nach ebd., S. 263.

97 Ebd., S. 262-264.

98 Beck, Macht-Räume, S. 86.

99 Ruth Frötschel, Mit Handkuss. Die Hand als Gegenstand des Zeremoniells am Wiener Hof im 17. und 18. Jahrhundert, in: Irmgard Pangerl/Martin Scheutz/Thomas Winkelbauer (Hrsg.), Der Wiener Hof im Spiegel der Zeremonialprotokolle (1652-1800). Eine Annäherung (Forschungen zur Landeskunde von Niederösterreich 31, Forschungen und Beiträge zur Wiener Stadtgeschichte 47), Innsbruck-Wien 2007, S. 337-356, hier S. 345. Gasser, Das Gesundheitsverhalten, S. 62

101 Brief Maria Theresias an Maria Beatrice, 23.8.1773, zit. nach Rothe, Die Mutter und die Kaiserin, S. 336. 
[die] Niederkunft [...] angeht, wollte ich unwissend bleiben, um besser gehorchen zu können." ${ }^{102}$

\subsection{Rituale nach der Geburt}

Nach der Entbindung bestimmte der Kaiser die Örtlichkeit und die Uhrzeit der Taufe. Diese fand meistens noch am selben Tag, spätestens aber am nächsten Tag statt. Organisiert wurde die Taufe vom Obersthofmeister ${ }^{103}$, der auch Botschafter, den päpstlichen Nuntius und andere Gäste zur Feierlichkeit einlud. Die Taufe fand in der Hofburg in der sogenannten Ritterstube statt, in Schönbrunn in einem der Vorzimmer. Ab 1755 wurde laut Hofzeremoniell sowohl in der Wiener Hofburg als auch im Schloss Schönbrunn die zweite Antecamera ${ }^{104}$ verwendet. Diese Regelung zur Änderung des Raumes in der Hofburg betraf allerdings nur mehr die letzten beiden Kinder, Maria Antonia und Maximilian Franz. ${ }^{105}$ Khevenhüller-Metsch vermerkte in seinem Tagebuch, dass die Taufe Maria Antonias am 3. November 1755 in der „Antikamera, weil die Ritterstube durch die neue Einrichtung der Zimmer zu klein geworden, geschehen ist" ${ }^{\text {"106. Der Saal }}$ wurde dafür jeweils mit Teppichen ausgestattet, auf einer Bühne befand sich ein Altar mit Baldachin und der traditionellen kaiserlichen Taufgarnitur. Vor dem Altar wurden die Kniebänke und Sessel für die Kaiserfamilie platziert. Zusätzlich wurden Reliquien aus der Schatzkammer geholt, die dem Säugling Kraft und Heil bringen sollten. Diese Reliquienverehrung während der Taufe wurde allerdings unter Maria Theresia beziehungsweise unter van Swieten abgeschafft. Durchgeführt wurde die Taufe durch den päpstlichen Nuntius in Anwesenheit der ganzen hohen Geistlichkeit. ${ }^{107}$ Während der Zeremonie wurde der Säugling von den Patinnen und Paten beziehungsweise dessen Stellvertreter*innen gehalten und der Kaiser nannte die Namen, die das Kind erhalten sollte. Der Kopf des Täuflings wurde dreimal mit Taufwasser, dem Jordanwasser beigemischt war, übergossen. ${ }^{108}$

Die Frömmigkeit der Dynastie der Habsburger*innen spiegelte sich auch in den Namensgebungen ihrer Kinder wider. Neben den Namen der Großeltern des Kindes, waren Namen der Jungfrau Maria und die anderer Heiligen eine beliebte Wahl. So trugen zehn Töchter Maria Theresias den Namen Maria. Die einzige Ausnahme stellte Johanna Gabriela dar. Thronfolger Joseph erhielt seinen Namen, da die schwangere Maria Theresia nach der Geburt von drei Töchtern zum heiligen Josef um den Erhalt eines Sohnes gebetet hatte. ${ }^{109}$ Bei einem weiteren Blick auf die Namensliste der Kinder fällt auf, dass

102 Brief Maria Theresias an Maria Beatrice, 23.8.1773, zit. nach Rothe, Die Mutter und die Kaiserin, S. 336-337.

103 Der Obersthofmeister hatte die gesamte Dienerschaft des Kaisers unter sich und hatte vor allem zeremonielle, repräsentative und organisatorische Aufgaben: Martin Mutschlechner, Im Vorhof der Macht - Die vier obersten Hofchargen, o. D., https://www.habsburger.net/de/kapitel/im-vorhof-der-macht-die-vier-obersten-hofchargen, eingesehen 28.2.2021.

104 Vorzimmer, Zeremoniensaal (Schloss Schönbrunn).

105 Beck, Macht-Räume, S. 85-87.

106 Tagebuch des kaiserlichen Obersthofmeister Johann Josef Khevenhüller-Metsch, 3.11.1755, zit. nach Maria Theresia, Familienbriefe, S. 158.

107 Vocelka/Heller, Die Lebenswelt, S. 274-276.

108 Kubiska, Geburten- und Taufzeremoniell am Wiener Hof, S. 497-500.

109 Weiss, Zur Herrschaft geboren, S. 41-43. 
Kinder oftmals wie ein bereits verstorbenes Kind benannt wurden. So gab es zweimal eine Maria Elisabeth und dreimal eine Maria Karolina.

Obwohl seit dem Konzil von Trient (1545-1563) lediglich ein Taufpate gestattet war, blieben Fürstenhäuser bei der Tradition von mehreren Patinnen und Paten. ${ }^{110}$ In erster Linie wurden hierfür Großeltern und nahe Verwandte ausgewählt. Bei Kindern des Kaisers kam der Wahl eine besondere politische Bedeutung zu, darum entschied man sich vor allem für Päpste und Monarchen. Eine Tradition, die besonders von Maria Theresia verfolgt wurde. Bei ihrem ersten Sohn und Thronfolger Joseph war unter anderem Papst Benedikt XIV. Pate. ${ }^{111}$ Für Leopold wurde die russische Zarin, für Johanna Gabriela der englische König und für Maria Karolina der französische König als Taufpate bestimmt. Maria Theresia wollte mit der Wahl der Pat*innen ihrer Kinder die Beziehungen zu den europäischen Höfen stärken. ${ }^{112}$

Nach dem Ritual der Taufe ertönte üblicherweise in der Kapelle das Te deum. ${ }^{113}$ Zeitgleich wurden drei Salven abgefeuert - eine jeweils am Beginn und Ende des Te deums und schließlich eine, als die Herrschaften aus der Ritterstube auszogen. ${ }^{114}$ Bis zu Maria Theresia geschah dies nur bei der Taufe von Erzherzögen. Die Königin änderte dies aber, sodass auch bei ihren Töchtern die Salutschüsse abgefeuert wurden. ${ }^{115}$ Da die Geschütze immer größer und lauter wurden, verbot van Swieten das Abfeuern jedoch, wenn sich der Säugling in unmittelbarer Nähe befand. ${ }^{116}$ Anschließend wurde das Kind wieder zur Mutter in das Gebärzimmer gebracht, wo es den Segen der beiden Eltern erhielt, bevor es in das eigene Appartement gebracht wurde. ${ }^{117}$ Bei den Tauffeierlichkeiten des Erzherzogs Josephs gab es allerdings einen Unterschied, denn als Thronfolger wurde er nach der Taufe mit einer weiteren Zeremonie in den Orden des Goldenen Vlieses aufgenommen. Joseph war damit einer von nur zwei Habsburgern, denen diese Aufnahmezeremonie direkt im Anschluss an die Tauffeierlichkeiten zuteil wurde. ${ }^{118}$

Bei den 16 Entbindungen Maria Theresias kam es zweimal zu einer Nottaufe. Bei der Entbindung des zehnten Kindes 1748 kam es bei der Geburt zu Komplikationen, ,,weillen das Kind nicht wohl gewendet und zuerst mit denen Füsselen gekommen"119. Gerard van Swieten und die Hebamme versicherten, dass sie das Kind mit der Nottaufe versehen hatten, dies wurde von der Hofgesellschaft allerdings angezweifelt. ${ }^{120}$ So lässt sich bei Khevenhüller-Metsch lesen, dass „ungehindert der Prothomedici Van Suiten und der Hebamme Versicherens, ville doch, ob es noch bei Leben die Tauff erhalten

110 Weiss, Die Österreicherin, S. 30.

111 Vocelka/Heller, Die Lebenswelt, S. 276-277.

112 Weiss, Zur Herrschaft geboren, S. 44.

113 Vocelka/Heller, Die Lebenswelt, S. 276.

114 Kubiska, Geburten- und Taufzeremoniell am Wiener Hof, S. 500-501.

115 Beck, Macht-Räume, S. 86.

116 Vocelka/Heller, Lebenswelt der Habsburger, S. 276.

117 Kubiska, Geburten- und Taufzeremoniell am Wiener Hof, S. 500.

118 Ebd., S. 501-502.

119 Tagebuch des kaiserlichen Obersthofmeister Johann Josef Khevenhüller-Metsch, 17.9.1748, zit. nach KhevenhüllerMetsch/Schlitter, Tagebuch des Fürsten Khevenhüller-Metsch, Bd. 2, S. 264.

120 Stollberg-Rilinger, Maria Theresia, S. 307-308. 
habe, zweiffeln wollen." ${ }^{21}$ Auch beim letzten Kind der Kaiserin kam es erneut zu einer Nottaufe, da sich der Bub ebenfalls nicht gedreht hatte. Er wurde von der Hebamme notgetauft, erholte sich aber im Laufe des Tages wieder, sodass die Taufe am Abend öffentlich wiederholt werden konnte. ${ }^{122}$

Die Kindbettzeit am Hofe wurde durch den ersten Messebesuch der Kaiserin und mit der Aussegnung der Wöchnerin und ihres Kindes beendet. ${ }^{123}$ Diese fand je nach Ort der Entbindung in der Loretokapelle der Augustinerkirche oder der Schlosskapelle Schönbrunns statt. Bei Totgeburten fand die Aussegnung in aller Stille in einer Kammerkapelle statt. ${ }^{124}$ Die Aussegnung beziehungsweise "der Hervorgang" Maria Theresias nach der Geburt Josephs wurde besonders feierlich begangen. Sechs Wochen nach der Entbindung fand in Wien eine Illumination - die normalerweise direkt nach der Geburt erfolgte - statt und die Bürger*innen demonstrierten ihre Anteilnahme. Maria Theresia befahl, diese Festlichkeiten auf ihren Hervorgang zu verschieben, damit sie selbst daran teilnehmen konnte. Zwei weitere Dinge bezüglich der üblichen Zeremonien und Gegebenheiten nach der Entbindung änderten sich mit Maria Theresia. Zum einen trug sie ab 1751 ihre Kinder selbst zur Aussegnung in die Kapelle. ${ }^{125}$ Zum anderen verzichtete sie während der Wochenbettzeit nicht mehr auf Gottesdienste. ${ }^{126}$ Im Anschluss an die Aussegnung wurde ein öffentliches Mahl abgehalten. ${ }^{127}$ Nach der Geburt Maria Antonias am Allerseelentag 1755 berichtete Khevenhüller-Metsch, „die Kaiserin wurde im Kindbett unwohl, und erst am 14. Dezember konnte der Hervorgang gefeiert werden"128.

\subsection{Verkündigung der Entbindung und anschließende Festivitäten}

Nach der Entbindung verkündete der Kaiser dem Volk die Geburt und es wurde ein Bote ausgesandt, um die Glocken im Stephansdom zu läuten. Kammerdiener wurden an befreundete Höfe geschickt, um ihnen die Geburt zu verkünden. ${ }^{129}$ Weiters wurden die jeweiligen Entbindungen im „Wiener Diarium“ veröffentlicht. Zur Entbindung selbst wurde wie in den Zeremonialprotokollen nur Datum und Uhrzeit berichtet, ob es ein Erzherzog oder eine Erzherzogin war und, ob die Geburt glücklich verlaufen war. Sogar bei Maria Karolina, die kurz nach der Geburt verstarb, wurde berichtet, dass es eine glückliche Entbindung gewesen war. ${ }^{130}$ Bei Johanna Gabriela sowie Maria Josepha war der Wortlaut der Meldung identisch. ${ }^{131}$

121 Tagebuch des kaiserlichen Obersthofmeister Johann Josef Khevenhüller-Metsch, 17.9.1748, zit. nach KhevenhüllerMetsch/Schlitter, Tagebuch des Fürsten Khevenhüller-Metsch, Bd. 2, S. 264.

122 Weiss, Zur Herrschaft geboren, S. 47

123 Weiss, Die Österreicherin, S. 20.

124 Vocelka/Heller, Die Lebenswelt, S. 276.

125 Bis zu diesem Zeitpunkt wurde das Kind von der ausgewählten Erzieherin des Kindes in die Kirche getragen: Stollberg-Rilinger, Maria Theresia, S. 313.

126 Ebd., S. 311-313.

127 Beck, Macht-Räume, S. 88.

128 Tagebuch des kaiserlichen Obersthofmeister Johann Josef Khevenhüller-Metsch, 3.11.1755, zit. nach Maria Theresia, Familienbriefe, S. 159.

129 Vocelka/Heller, Die private Welt, S. 28.

130 Wienerisches Diarium, 18.9.1748, S. 7.

131 Siehe dazu: Wienerisches Diarium, 7.2.1750, S. 7; Wienerisches Diarium, 20.3.1751, S. 7. 
Ein weiteres Ritual um die Zeit der Entbindung war die „päpstliche Windelpräsentation", bei der die Geschenke des Papstes in eine Windel eingewickelt präsentiert wurden. Diese Geschenke wurden nur katholischen, männlichen Thronfolgern zuteil. Ein außergewöhnlicher Fall war hier Maria Theresias erstgeborener Sohn, der spätere Kaiser Joseph II.,Seine“ Windelpräsentation fand nämlich erst statt, als er bereits im sechsten Lebensjahr war, was auf das gespannte Verhältnis zwischen Österreich und dem Vatikan zur Zeit seiner Geburt zurückzuführen ist. ${ }^{132}$ Diese Windeln wurden im Audienzzimmer ausgestellt, damit sie vom Hofstaat betrachtet werden konnten. Auch eine öffentliche Präsentation der Mutterschaft der Fürstin wurde durchgeführt: An den Fürstenhöfen war es üblich, dass die Fürstin nach der Geburt für Glückwunschbekundungen von Gesandten „Öffentlich“ im Bett lag. Maria Theresia hielt von dieser Form des Rituals offenbar nicht viel und präsentierte sich selbst nur dreimal im Paradebett liegend, ansonsten tat sie dies immer stehend. Nach der Geburt Josephs wurde ihr Bett allerdings zur öffentlichen Schau zur Verfügung gestellt. ${ }^{133}$

Wurde ein Prinz geboren, wurde drei Tage lang eine große Gala am Wiener Hof gefeiert. Bei einer Prinzessin wurde am ersten Tag eine große Gala gefeiert und an den darauffolgenden zwei Tagen eine kleine Gala. ${ }^{134}$ Auch die Bevölkerung der Residenzstadt Wien feierte die Geburt mit Illuminationen und Tanz in den Straßen, in anderen Städten wurde in bescheidenerem Ausmaß zelebriert. Für solche Festivitäten wurden vom Kaiserhaus Geld, Semmeln, Krapfen und Wein gespendet. Im Jahre 1743, als mit Joseph der Thronfolger geboren wurde, wurde ein eigenes Festschießen veranstaltet. Maria Theresia verschob diese Feiern teilweise zeitlich nach hinten, sodass sie nach ihrer Aussegnung selbst daran teilnehmen konnte. ${ }^{135}$

\section{Fazit}

Das Leben Maria Theresias war der Öffentlichkeit gewidmet. Der Körper einer Herrscherin beziehungsweise der Frau eines Herrschers war ein Politikum und gehörte nicht ihr allein. Dieser Körper war zuständig dafür, dass die Dynastie weiterhin bestand. Damit lässt sich auch erklären, dass die Verkündung der Schwangerschaft, Aderlässe und Geburten der breiten Öffentlichkeit im „Wienerischen Diarium“ bekannt gemacht wurden. Umso erstaunlicher ist es, dass in den Zeitungsbeiträgen, den Tagebucheinträgen Khevenhüller-Metschs, den Zeremonialprotokollen und den Briefen der Herrscherin keine genaueren Aufzeichnungen zum Entbindungsvorgang bis auf Datum, Zeit, Ort und den Ausgang der Entbindung aufgeschrieben wurden. Letzten Endes blieb der Körper der Herrscherin doch bis zu einem bestimmten Grad privat. Daher ist es auch schwierig, die „adeligen“ Entbindungen Maria Theresias mit denen in anderen Bevölkerungsschichten zu vergleichen. Die Pflicht einer adeligen Frau, Kinder zu bekommen, erfüllte Maria Theresia vollends. Es lässt sich jedoch im Laufe der Zeit ein Umdenken bei ihren

\footnotetext{
132 Vocelka/Heller, Die Lebenswelt, S. 276.

133 Stollberg-Rilinger, Maria Theresia, S. 312-313.

134 Beck, Macht-Räume, S. 86.

135 Vocelka/Heller, Die Lebenswelt, S. 277.
} 
Schwangerschaften feststellen. Während sie die ersten noch leicht hinnahm, fielen ihr diese mit zunehmendem Alter sichtlich schwerer. Wünschte sie sich nach der Geburt Josephs direkt wieder schwanger zu sein, so hoffte sie während der Schwangerschaft ihres zehnten Kindes, dass diese die letzte sein würde.

Der Wiener Hof des 18. Jahrhunderts war stark gekennzeichnet durch das Zeremoniell und die damit verbundenen Rituale. Auch die Geburten und Entbindungen waren durch sie bestimmt. Um auf alle Eventualitäten rituell vorbereitet zu sein, wurden vor der Niederkunft eigene Sitzungen zum Ablauf der Zeremonien rund um die Geburt abgehalten. Bei Maria Theresia lässt sich eine Befolgung des Zeremoniells erkennen, gleichzeitig durchbrach sie es aber, indem sie Neuerungen einführte. So entschied sie sich ab 1747 ihren Entbindungsort in den Sommermonaten nach Schönbrunn zu verlegen, anstatt dafür zurück in die Hofburg zu fahren. Zugunsten ihrer Töchter änderte sie das Zeremoniell, sodass diese bei deren Taufe ebenfalls Salutschüsse bekamen und nicht mehr nur die Erzherzöge. Die wohl größte Veränderung war der Einzug des Arztes Gerard van Swieten in das Gebärzimmer und der gleichzeitige Auszug der Hofdamen. Besonders lässt sich diesbezüglich aber die Bedeutung von Zeremoniell erkennen, denn dieser Auszug musste durch einen vorgezogenen Handkuss kompensiert werden, um die Ehre der Damen aufrecht zu erhalten. Eine Konstante im Gebärzimmer gab es aber: die Hebamme. Hier ist auffällig, dass diese nie namentlich erwähnt wurde. Das hängt damit zusammen, dass die Hebamme nicht als Person wichtig war, sondern lediglich ihre Funktion.

Bei den Ritualen rund um Schwangerschaften und Entbindungen ist trotz einiger Änderungen bei späteren Entbindungen ein gewisser roter Faden erkenntlich. Der vorgesehene Ablauf begann mit der öffentlichen Verkündung der Schwangerschaft, indem die Herrscherin in einer Sänfte zur Kirche getragen wurde. Daraufhin wurden in den Ländereien der Herrscherin Gebete für eine glückliche Schwangerschaft und Entbindung angesetzt. Während dieser Zeit wurde an der Schwangeren dreimal ein Aderlass durchgeführt, der ebenfalls ein öffentliches Ereignis war. Beim Eintreten der Wehen wurde in den Kapellen das Allerheiligste ausgesetzt und die Hofgesellschaft versammelte sich. Nach der Entbindung verkündete der Kaiser die Geburt eines Sohnes oder einer Tochter und bestimmte einen Zeitpunkt für die Taufe, die ebenfalls einem Hofzeremoniell folgte. Die Wochenbettzeit Maria Theresias war meist kürzer als bei anderen Adeligen, da sie als regierende Herrscherin eine besondere Rolle einnahm. Diese Überschneidung zwischen Herrscherin und Wöchnerin könnte in zukünftigen Forschungen noch näher betrachtet werden. Beendet wurde die Phase der Schwangerschaft, Entbindung und Wochenbettzeit durch die Aussegnung der Königin in der Kirche, woraufhin meist bald die nächste Schwangerschaft folgte und der Kreislauf von Neuem begann. 


\section{Quellen und Quelleneditionen}

Birkenbihl, Wolf H., Maria Theresia - Monarchin, Mutter und Mensch. Ihr Leben und ihre Zeit in Briefen und Augenzeugenberichten, Baden-Baden 2017.

Christoph, Paul (Hrsg.), Maria Theresia und Marie Antoinette. Der geheime Briefwechsel, Darmstadt 2017.

Khevenhüller-Metsch, Rudolf/Schlitter, Hanns, (Hrsg.), Aus der Zeit Maria Theresias. Tagebuch des Fürsten Johann Josef Khevenhüller-Metsch. Kaiserlichen Obersthofmeisters. 1742-1776, Bd. 1, Leipzig-Wien 1907.

Dies. (Hrsg.), Aus der Zeit Maria Theresias. Tagebuch des Fürsten Johann Josef Khevenhüller-Metsch. Kaiserlichen Obersthofmeisters. 1742-1776, Bd. 2, Leipzig-Wien 1908.

Maria Theresia, Familienbriefe. Mit einem biographischen Anhang (Die Fünfzig Bücher 3), Berlin-Wien 1916.

Rothe, Carl (Hrsg.), Die Mutter und die Kaiserin. Briefe der Maria Theresia an ihre Kinder und Vertraute, Berlin 1987.

Walter, Friedrich (Hrsg.), Maria Theresia. Briefe und Aktenstücke in Auswahl (Ausgewählte Quellen zur Deutschen Geschichte der Neuzeit 12), Darmstadt 1968.

Wienerisches Diarium, 23.11.1740, 15.4.1747, 13.4.1748, 18.9.1748, 7.2.1750, 20.3.1751, 22.7.1752.

\section{Literatur}

Banakas, Anne-Sophie, Die zwei Körper der Herrscherin. Der politische und der natürliche Körper in den Porträts von Maria Theresia (1740-1780), in: Mitteilungen des Instituts für Österreichische Geschichtsforschung 126 (2018), Heft 1, S. 73-109.

Barth-Scalmani, Gunda, "Freundschaftlicher Zuruf eines Arztes an das Salzburgische Landvolk": Staatliche Hebammenausbildung und medizinische Volksaufklärung am Ende des 18. Jahrhunderts, in: Jürgen Schlumbohm u. a. (Hrsg.), Rituale der Geburt. Eine Kulturgeschichte, München 1998, S. 102-118.

Beck, Marina, Macht-Räume Maria Theresias. Funktion und Zeremoniell in ihren Residenzen, Jagd- und Lustschlössern (Kunstwissenschaftliche Studien 189), BerlinMünchen 2017.

Egghardt, Hanne, Maria Theresias Männer. Ihre Lieben, ihre Ratgeber und die Stützen ihres Throns, Wien 2015.

Ehalt, Hubert Ch., Ausdrucksformen absolutistischer Herrschaft. Der Wiener Hof im 17. und 18. Jahrhundert (Sozial- und Wirtschaftshistorische Studien 14), Wien 1980.

Frötschel, Ruth, Mit Handkuss. Die Hand als Gegenstand des Zeremoniells am Wiener Hof im 17. und 18. Jahrhundert, in: Irmgard Pangerl/Martin Scheutz/Thomas Winkelbauer (Hrsg.), Der Wiener Hof im Spiegel der Zeremonialprotokolle (1652-1800). Eine 
Annäherung (Forschungen zur Landeskunde von Niederösterreich 31, Forschungen und Beiträge zur Wiener Stadtgeschichte 47), Innsbruck-Wien 2007, S. 337-356.

Gasser, Monika Gertraud, Das Gesundheitsverhalten von Herrscherinnen und Herrschern des 18. Jahrhunderts im Vergleich. Dargestellt am Beispiel von Maria Theresia, Friedrich dem Großen, Marie Antoinette und Joseph II., Dipl. Innsbruck 2005.

Gélis, Jacques, Die Geburt. Volksglaube, Rituale und Praktiken von 1500-1900, übersetzt von Clemens Wilhelm, München 1989.

Gerabek, Werner E., Swieten, Gerard van (Gerhard von), in: Neue Deutsche Biographie, Bd. 25, Berlin 2013, S. 729-730.

Hilber, Marina, Institutionalisierte Geburt. Eine Mikrogeschichte des Gebärhauses, Bielefeld 2012

Horn, Sonia, „... dann mit meiner Hebammerey ich vill mehr gewinnen kann, alß mein Mann mit seiner Doctorey.." Wiener Hebammen 1700-1750, Dipl, Wien 1995.

Huber, Heinz, Pioniere aus Medizin- und Geschichtswissenschaften in Österreich. Ausgewählte Biographien aus über zwei Jahrhunderten, Innsbruck 2017.

Kubiska, Irene, Und ist wegen dieser so glücklich- und trostreichen Geburth ein allgemeines Frolockhen und grosse Freydt gewesen. Das Geburten- und Taufzeremoniell am Wiener Hof im Zeitraum von 1652 bis 1800, in: Irmgard Pangerl/Martin Scheutz/ Thomas Winkelbauer (Hrsg.), Der Wiener Hof im Spiegel der Zeremonialprotokolle (1652-1800). Eine Annäherung (Forschungen zur Landeskunde von Niederösterreich 31, Forschungen und Beiträge zur Wiener Stadtgeschichte 47), Innsbruck-Wien 2007, S. 493-527.

Labouvie, Eva, Selbstverwaltete Geburt. Landhebammen zwischen Macht und Reglementierung (17.-19. Jahrhundert), in: Geschichte und Gesellschaft 18 (1992), Heft 4, S. 447-506.

Dies., Andere Umstände. Eine Kulturgeschichte der Geburt, Köln 1998.

Dies., Beistand in Kindsnöten. Hebammen und weibliche Kultur auf dem Land (15501910) (Geschichte und Geschlechter 29), Frankfurt-New York 1999.

Mutschlechner, Martin, Im Vorhof der Macht - Die vier obersten Hofchargen, O. D., https://www.habsburger.net/de/kapitel/im-vorhof-der-macht-die-vier-oberstenhofchargen, eingesehen 28.2.2021.

Pawlowsky, Verena, Trinkgelder, Privatarbeiten, Schleichhandel mit Ammen. Personal und Patientinnen in der inoffiziellen Ökonomie des Wiener Gebärhauses (1784-1908), in: Jürgen Schlumbohm u. a. (Hrsg.), Rituale der Geburt. Eine Kulturgeschichte, München 1998, S. 206-220.

Pethes, Nicolas, Kulturwissenschaftliche Gedächtnistheorien zur Einführung, Hamburg 2008. 
Schlumbohm, Jürgen, Kinderstuben. Wie Kinder zu Bauern, Bürgern, Aristokraten wurden, 1700-1850, München 1983.

Schnettger, Matthias, Rezension zu: Barbara Stollberg-Rilinger, Maria Theresia. Die Kaiserin in ihrer Zeit, München 2017, in: sehepunkte 17 (2017), Heft 9, http://www. sehepunkte.de/2017/09/30136.html, eingesehen 14.2.2020.

Stollberg-Rilinger, Barbara, Rituale (Historische Einführungen 16), Frankfurt am Main 2013.

Dies., Maria Theresia. Die Kaiserin in ihrer Zeit, München 2017.

Vocelka, Karl/Heller, Lynne, Die Lebenswelt der Habsburger. Kultur- und Mentalitätsgeschichte einer Familie, Graz-Wien-Köln 1997.

Ders./Dies., Die private Welt der Habsburger. Leben und Alltag einer Familie, GrazWien-Köln 1998.

Walter, Friedrich (Hrsg.), Maria Theresia. Briefe und Aktenstücke in Auswahl (Freiherrvom-Stein-Gedächtnisausgabe. Ausgewählte Quellen zur Deutschen Geschichte der Neuzeit 12), Darmstadt 1968.

Weiss, Sabine, Die Österreicherin. Die Rolle der Frau in 1000 Jahren Geschichte, GrazWien-Köln 1996.

Dies., Zur Herrschaft geboren. Kindheit und Jugend im Haus Habsburg von Kaiser Maximilian I. bis Kronprinz Rudolf, Innsbruck 2008.

Sabrina Schober ist Masterstudentin der Geschichtswissenschaften an der Universität Innsbruck. sabrina.schober@student.uibk.ac.at

\section{Zitation dieses Beitrages}

Sabrina Schober, Hofzeremoniell und Gebärstuhl. Zur Bedeutung von Ritualen bei den Entbindungen Maria Theresias (1717-1780), in: historia.scribere 13 (2021), S. 233-256, [http://historia.scribere.at], eingesehen 22.6.2021 (=aktuelles Datum). 